\title{
Methodologies of chattering attenuation in sliding mode controller
}

\begin{abstract}
Uncertain or complicated systems are difficult to control. Modeling the system uncertainties is an especial topics in most of engineering field. On the other hand, since system has uncertainty, design stable and robust controller is crucial importance in control engineering. To solve this challenge nonlinear control technique is the best choice. Sliding mode control is one important type of robust control. Model imprecision may come from actual uncertainty about the plant or from a purposeful simplification of the system's dynamics. Modeling inaccuracies can cause strong adverse effects on the control design of nonlinear systems. For the class of systems to which it applies, sliding mode controller design provides a systematic approach to the problem of maintaining stability and consistent performance in the face of modeling imprecision. However, sliding mode controller is a robust and stable controller but it has an important challenge called, chattering phenomenon. This research focuses on the comparative between three methods to eliminate/reduce the chattering.
\end{abstract}

Keyword: Variable structure controller; Sliding surface slope; Chattering phenomenon; Boundary layer methodology; PD parallel chattering attenuation; Intelligent chattering elimination 